\title{
ARMAZENAMENTO DE PITANGAS SOB ATMOSFERA MODIFICADA E REFRIGERAÇÃO: II - QUALIDADE E CONSERVAÇÃO PÓS-COLHEITA ${ }^{1}$
}

\author{
ADRIANA FERREIRA DOS SANTOS², SILVANDA DE MELO SILVA3, REJANE MARIA NUNES MENDONÇA4, \\ HELOÍSA ALMEIDA CUNHA FILGUEIRAS ${ }^{5}$
}

\begin{abstract}
RESUMO - Este trabalho teve como objetivo avaliar o efeito de atmosfera modificada (AM) na conservação pós-colheita de pitangas colhidas nos estádios de maturação e início da pigmentação (IP), vermelho-alaranjado (VA) e vermelho predominante (VP) e armazenadas a 10 e a $14{ }^{\circ} \mathrm{C}(90 \pm$ $1 \% \mathrm{UR})$ e condições ambientes $\left(23 \pm 2{ }^{\circ} \mathrm{C}\right.$ e $\left.85 \pm 2 \% \mathrm{UR}\right)$. $\mathrm{O}$ uso de $\mathrm{AM}$ associada à refrigeração resultou em menores perdas de massa, incidência de fungo e enrugamento. A AM também permitiu um aumento de quatro dias na vida útil pós-colheita, mantendo a qualidade acima do limite de aceitação durante oito dias, para pitangas do estádio VA mantidas sob refrigeração. Em conjunto, pitangas colhidas no estádio de maturação vermelho-alaranjado apresentaram melhor potencial de armazenamento, quando mantidas sob atmosfera modificada a $10^{\circ} \mathrm{C}$.
\end{abstract}

Termos para indexação: Eugenia uniflora, estádio de maturação, podridão, enrugamento, aparência geral.

\section{STORAGE OF SURINAME CHERRY UNDER MODIFIED ATMOSPHERE AND REFRIGERATION: QUALITY AND POSTHARVEST CONSERVATION}

\begin{abstract}
The aim of this work was to evaluate the effect of modified atmosphere (MA) on postharvest conservation of Suriname cherry harvested in the maturity stages: pigment initiation (PI), red-orangish (RO) and predominant red (PR), and stored at $10,14^{\circ} \mathrm{C}(90 \pm 1 \% \mathrm{RH})$, and room temperature $\left(23 \pm 2{ }^{\circ} \mathrm{C}\right.$ and $\left.85 \pm 2 \% \mathrm{RH}\right)$. The use of MA associated with refrigeration resulted in lower mass loss, fungi incidence, and fruit shrinkage. The MA also allowed a four-day increase in postharvest life, maintaining the general quality above acceptance limit during eight days, for Suriname cherries of RO maturity stage, kept under refrigeration. Collectively, Suriname cherries harvested at the maturity stage red-orangish presented the best storage potential, when kept under modified atmosphere at $10{ }^{\circ} \mathrm{C}$.
\end{abstract}

IndexTerms: Eugenia uniflora, maturity stage, decay, fruit shrinkage, appearance.

\section{INTRODUÇÃO}

A pitanga (Eugenia uniflora L.) é um fruto delicado e de elevada perecibilidade, suscetível a injúrias mecânicas, deterioração fisiológica, excessiva perda de água e podridão, dificultando seu armazenamento e comercialização. O pronto resfriamento de pitanga pode retardar mudanças indesejáveis de qualidade e aumentar a sua vida útil pós-colheita (Melo et al., 2000; Santos et al., 2003); no entanto, a sensibilidade a baixas temperaturas pode resultar em danos pelo frio, restringindo o seu tempo de armazenamento (Morris, 1982). A atmosfera modificada vem sendo utilizada com o objetivo de minimizar as perdas e prolongar a vida útil pós-colheita de frutos e hortaliças (Kays, 1997), estabelecendo uma composição gasosa diferente da do ar, pela redução do nível de $\mathrm{O}_{2}$ e elevação de $\mathrm{CO}_{2}$ no interior da embalagem, que pode resultar na redução da atividade metabólica do produto (Beaudry, 2004). A curta vida útil da pitanga torna o emprego de atmosfera modificada uma boa opção para o seu armazenamento (Santos et al., 2002), minimizando a infecção fúngica (Jaime et al., 2001), podendo estender substancialmente seu período de comercialização (Saez et al., 1999).

O objetivo deste trabalho foi avaliar a influência do estádio de maturação e utilização de atmosfera modificada sobre a qualidade e conservação pós-colheita de pitanga armazenada a $10^{\circ} \mathrm{C}$ e $(90 \pm 1 \% \mathrm{UR})$, $14{ }^{\circ} \mathrm{C} \mathrm{e}(90 \pm 1 \%$ UR $)$ e sob condições ambientes $\left(23 \pm 1{ }^{\circ} \mathrm{C}\right.$ e $85 \pm 2 \%$ UR $)$.

\section{MATERIAL E MÉTODOS}

Pitangas foram colhidas do Pomar da Embrapa Algodão, em Campina Grande, Estado da Paraíba, antes das 8 horas da manhã, nos estádios de maturação onde a epiderme dos frutos achavam-se no início da pigmentação vermelha (IP); vermelho-alaranjado (VA); predominantemente vermelho (VP). Os frutos foram transportados para o laboratório em caixas isotérmicas, imersos por 10 minutos em solução de hipoclorito de sódio a $0,5 \%$ e, em seguida, enxaguados com água (10 ppm de cloro livre) e secos ao ar. Aproximadamente $300 \mathrm{~g}$ de frutos foram acondicionados em bandejas de poliestireno com dimensões 250 x 150 x $25 \mathrm{~mm}$, sendo metade do total das bandejas envolvida com filme de cloreto de polivinila (PVC) flexível de 12 ìm de espessura para a modificação da atmosfera. As bandejas foram armazenados a $10^{\circ} \mathrm{Ce}(90$ $\pm 1 \% \mathrm{UR})$, a $14^{\circ} \mathrm{C} \mathrm{e}(90 \pm 1 \% \mathrm{UR})$ e à temperatura ambiente $\left(23 \pm 1^{\circ} \mathrm{C}\right.$ e 85 $\pm 2 \%$ UR). As análises realizadas foram perda de massa (\%), incidência de fungos, enrugamento (\%) e aparência geral: determinada em escala de 1 a 9 (1- Inaceitável; 3 - Ruim; 5-Regular; 7- Bom; 9 -Excelente). A incidência de infestações fúngicas e o enrugamento foram avaliados de forma independente, mediante o aparecimento de sintomas característicos, em escala de 1 a $9(1=$ sem infestações, $0 \%$ de enrugamento; $2=1$ a $5 \% ; 3=6$ a $15 \% ; 4=16$ a $30 \% ; 5=31$ a $45 \%$; $6=46$ a $60 \% ; 7=61$ a $75 \% ; 8=76$ a $85 \%$, e $9 \geq 85 \%$ ). Os fungos que se desenvolveram no decorrer do armazenamento, foram identificados no Departamento de Fitotecnia do CCA/UFPB/AREIA. O grau 4 da escala caracteriza o limite de aceitação do fruto in natura pelo consumidor. $\mathrm{O}$ experimento foi instalado em parcela subdividida no tempo, em esquema fatorial $(3 \times 2 \times 5)$, com três repetições. Os fatores estudados foram três estádios de maturação (IP, VA, VP), modificação da atmosfera em dois níveis (AA (atmosfera ambiente) e AM (atmosfera modificada)), e períodos de armazenamento. As avaliações subjetivas foram transformadas por $\sqrt{(X+1)}$. A partir dos resultados das análises de variância e verificando-se a interação entre os fatores, o tempo foi desdobrado dentro de cada tratamento, e os resultados submetidos à análise de regressão, sendo consideradas equações de até $2^{\circ}$ grau. $\mathrm{O}$ coeficiente de determinação mínimo para utilização das curvas foi de 0,60. Para casos em que a interação entre os fatores não foi significativa, os valores foram representados pelas médias, sem o ajuste de curvas.

\section{RESULTADOS E DISCUSSÃO}

O uso de atmosfera modificada (AM) proporcionou redução significativa $(P \leq 0,01)$ da perda de massa em pitangas quando comparada à atmosfera ambiente (AA), independentemente da

\footnotetext{
${ }^{1}$ (Trabalho 173/2004). Recebido: 01/11/2004. Aceito para publicação: 08/02/2006. Parte do trabalho de Dissertação do Mestrado em Agronomia do primeiro autor. ${ }^{2}$ Eng. Agr. M.Sc em Agronomia, Aluno de Doutorado do Programa de Pós-Graduação em Agronomia, CCA/UFPB, Caixa Postal 04, adre.santos@bol.com.br;

${ }^{3}$ Prof. Ph.D., DCFS/CCA/UFPB, Caixa Postal 04, CEP.: 58397-970, Areia, PB, silvasil@cca.ufpb.br;

${ }^{4}$ Prof. D. Sc., DF/CCA/UFPB, Areia, PB; rejane@cca.ufpb.br;

${ }^{5}$ Pesq. Dr., Embrapa-CNPAT, Caixa Postal 3711, Fortaleza-CE, heloisa@cnpat.embrapa.br.
} 


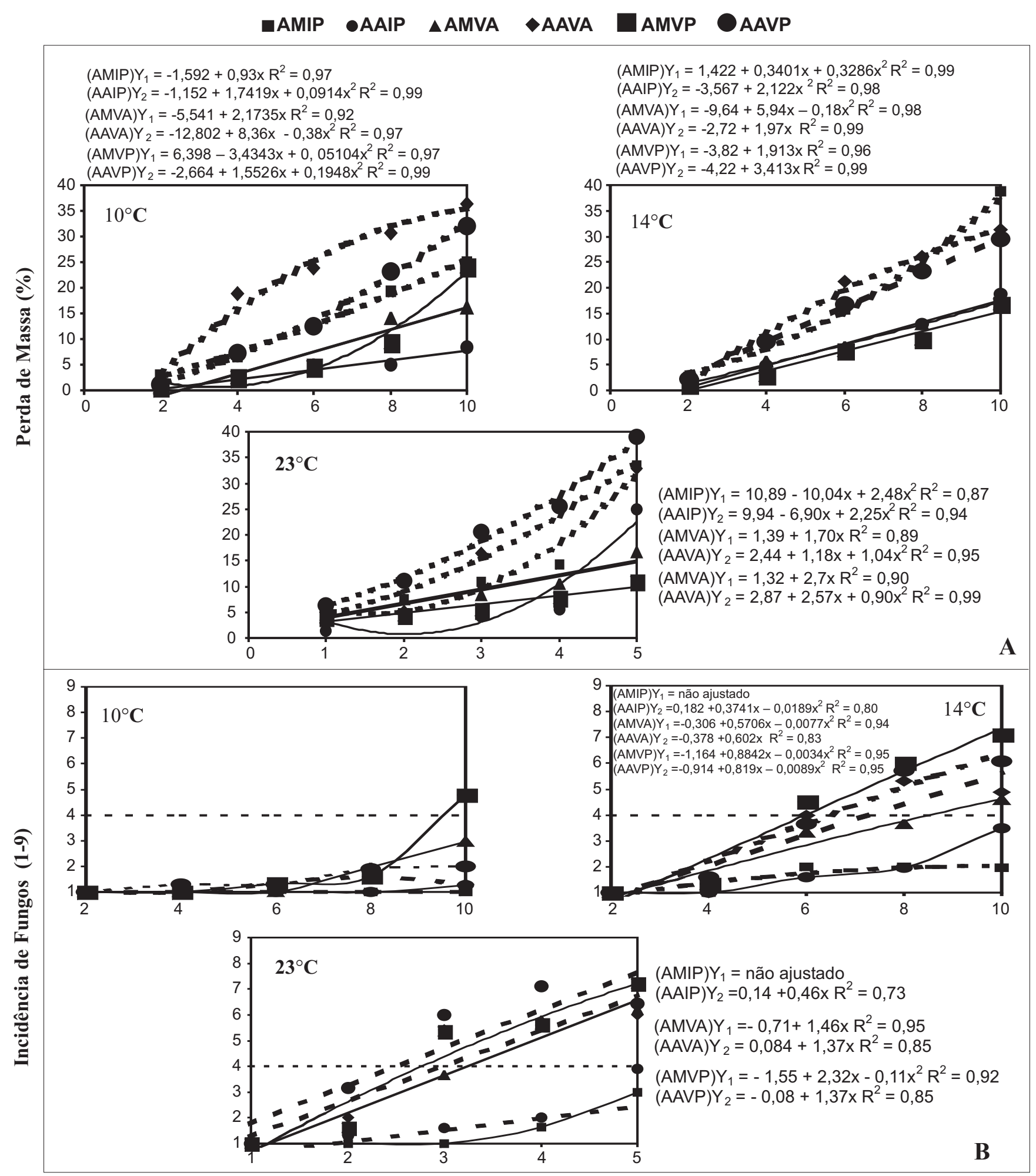

Período pós-colheita (Dias)

FIGURA 1 - Perda de massa (A) e Incidência de fungos (B) em pitanga nos estádios de maturação-início de pigmentação (IP), vermelho-alaranjado (VA) e vermelho predominante (VP) durante o armazenamento sob atmosfera modificada (AMY $\longrightarrow$ ) e ambiente $($ AAY $-\longrightarrow)$ a 10 e a $14^{\circ} \mathrm{C}$ e à temperatura ambiente $\left(23 \pm 2^{\circ} \mathrm{C}\right)$. O limite 4 indica o nível de aceitação do fruto in natura pelo consumidor de pitanga.

temperatura de armazenamento (Fig. 1A). Pitangas armazenadas em estádios de maturação mais avançados, tais como o predominantemente vermelho (VP), tenderam a apresentar maior perda de massa para frutos mantidos sob AM a $10^{\circ} \mathrm{C}$, certamente em decorrência da maior suculência dos frutos. O efeito da AM na redução da perda de massa dos frutos armazenados sob refrigeração $\left(14\right.$ e $\left.10{ }^{\circ} \mathrm{C}\right)$ é atribuído à redução da temperatura e associado à $\mathrm{AM}$, que mantém a UR elevada no interior da embalagem (Ben-Yehoshua, 1987). A perda de massa após 5 dias a $23^{\circ} \mathrm{C}$ e 10 dias sob refrigeração resultou em murchamento pronunciado nos frutos mantidos sob AA, influenciando negativamente a qualidade dos frutos. Pitangas do estádio IP mantidas a $10^{\circ} \mathrm{C}$ e AM apresentaram menor perda de massa quando comparadas às armazenadas a $14{ }^{\circ} \mathrm{C}$ e 23 ${ }^{\circ} \mathrm{C}$, o que pode ser explicado pelo menor DPV (déficit de pressão de vapor), proporcionado pela temperatura mais baixa, aliada à UR mais alta naquela condição de armazenamento (Grierson e Wardowsk, 1978).

A podridão pós-colheita é responsável por significativo percentual de perdas (Ahmadi et al., 1999). A incidência de fungos aumentou significativamente $(\mathrm{P} \leq 0,05)$ com o período de armazenamento para temperatura ambiente e $14{ }^{\circ} \mathrm{C}$, exceto para $10{ }^{\circ} \mathrm{C}$, onde não foi significativa. A modificação da atmosfera resultou em maior incidência de fungos ao final do armazenamento para frutos de maturidade mais avançada (Fig. 1B). Este aumento possivelmente foi devido à formação de uma atmosfera saturada de $\mathrm{CO}_{2}$ e deficiente em $\mathrm{O}_{2}$, favorecendo a proliferação de agentes patogênicos tolerantes a estas atmosferas, como o também observado em pimentão por Meier et al. (1995). O estádio IP, nas três temperaturas avaliadas, apresentou menor incidência de fungos 
quando comparado aos demais estádios de maturação. Isso possivelmente ocorreu devido à presença de substâncias de defesa, tais como alcalóides e fenólicos, que tornam os frutos mais resistentes. Os fungos que se desenvolveram com maior incidência durante o armazenamento, foram identificados como Clasdoporium cladosporioides, Colletotrichum gloeosporioides, Pestalotia sp. e Nigospora $\mathrm{sp}$. Os frutos armazenados a $10{ }^{\circ} \mathrm{C}$ apresentaram baixa incidência de fungos, com tendência de aumento a partir do $8^{\circ}$ dia de armazenamento para os frutos mantidos sob AM. A mais baixa incidência de fungos observada a $10{ }^{\circ} \mathrm{C}$, no entanto, pode ser atribuída à possibilidade de essa temperatura ter inibido a germinação dos esporos presentes, sobretudo em frutos mantidos sob AA. Os frutos armazenados sob condições ambientes sob AM apresentaram maior incidência de fungos, sendo o aumento da umidade dentro da embalagem fator determinante no desenvolvimento de microrganismos.

Verificou-se menor grau de enrugamento nos frutos sob AM

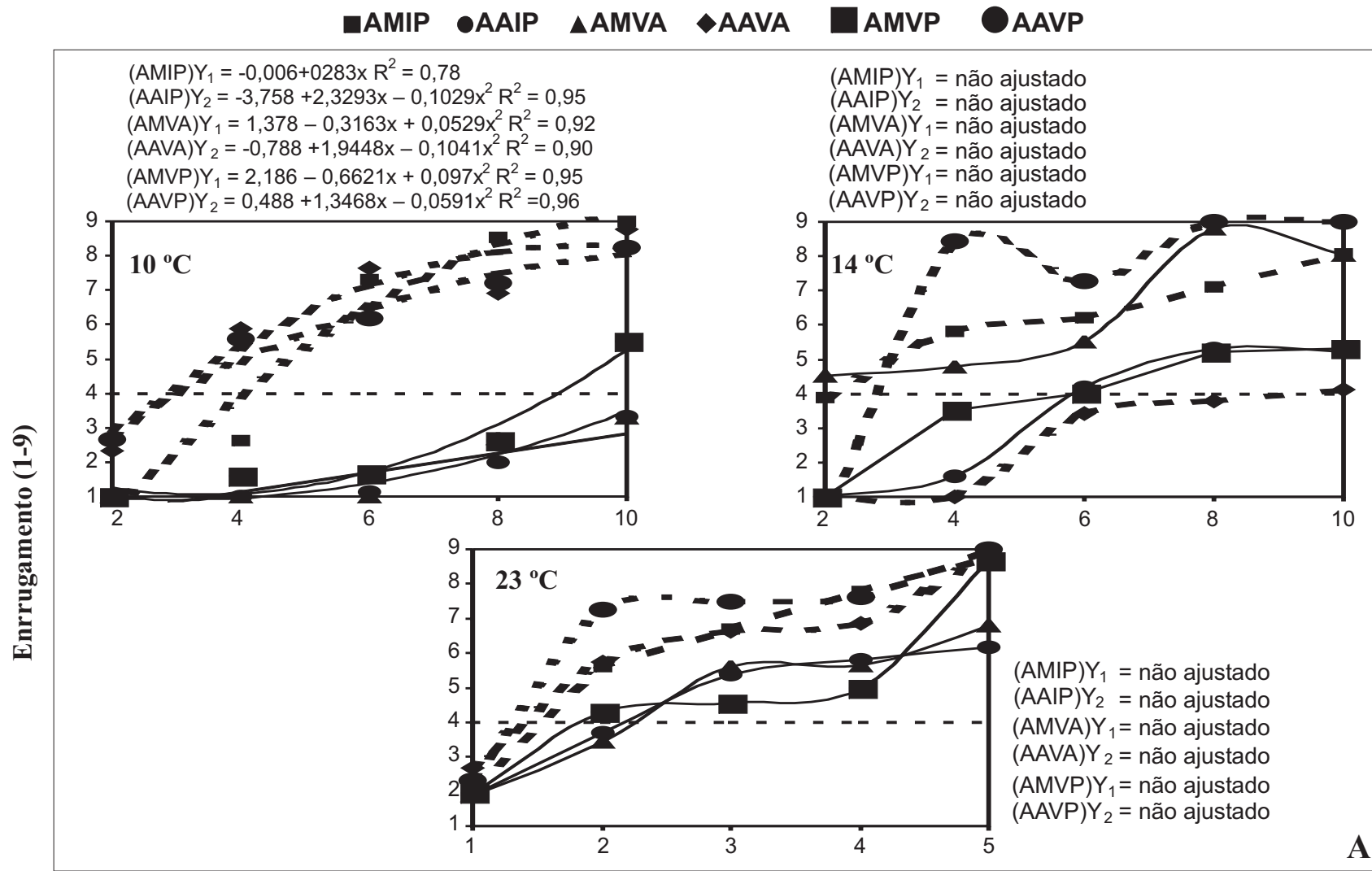

$\left(\right.$ AMIP) $Y_{1}=10,092-0,9613 x+0,0354 x^{2} R^{2}=0,95$ (AAIP) $Y_{2}=4,952+0,3326 x-0,0682 x^{2} R^{2}=0,97$

(AMVA) $Y_{1}=9,034-0,8345 x+0,0237 x^{2} R^{2}=0,93$ $($ AAVA $) Y_{2}=7,498-1,1355 x+0,0512 x^{2} R^{2}=0,92$ $\left(\right.$ AMVP) $Y_{1}=6,69-0,352 x R^{2}=0,61$

$\left(\right.$ AAVP) $Y_{2}=4,432-0,125 x-0,0175 x^{2} R^{2}=0,97$
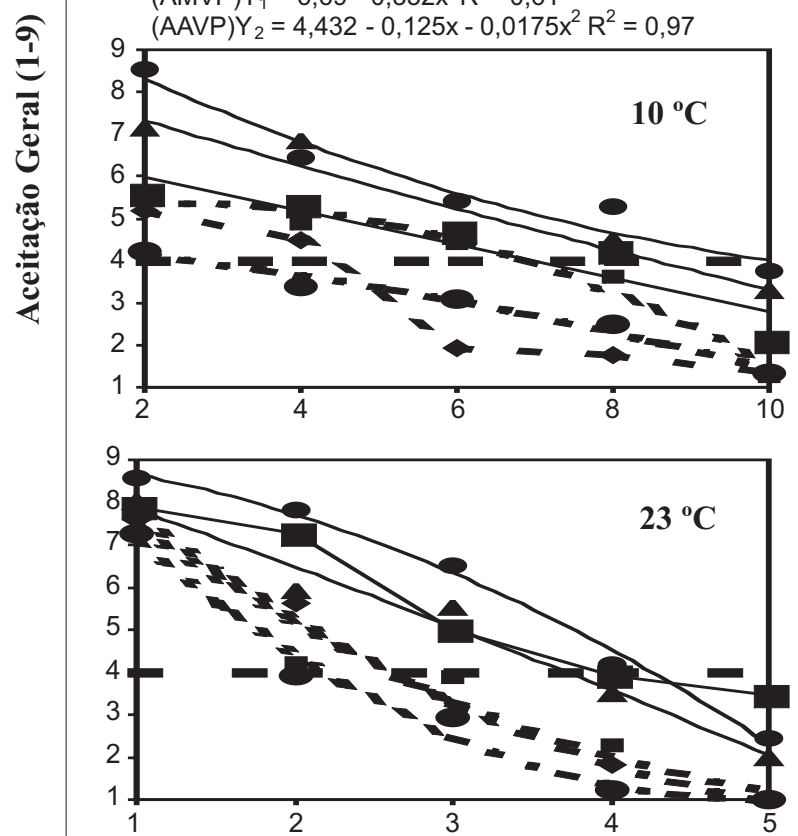

$\left(\right.$ AMIP) $Y_{1}=-7,464-0,094 x-0,042 x^{2} R^{2}=0,98$ (AAIP) $Y_{2}=5,29-0,4155 x R^{2}=0,90$

$\left(\right.$ AMVA) $Y_{1}=8,79-0,6245 x+0,0037 x^{2} R^{2}=0,99$

$\left(\right.$ AAVA) $Y_{2}=5,27-0,433 x R^{2}=0,86$

$(A M V P) Y_{1}=6,89-0,6665 \times R^{2}=0,89$ $(A A V P) Y_{2}=$ não ajustado

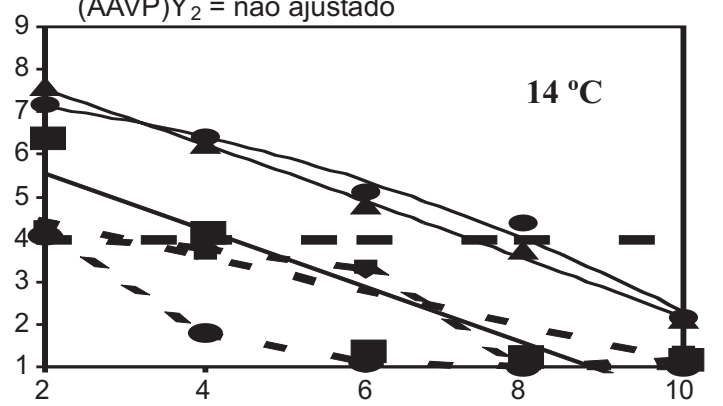

(AMIP) $Y_{1}=9,166-0,2937 x-0,2157 x^{2} R^{2}=0,99$

$\left(\right.$ AAIP) $Y_{2}=10,236-3,0376 x+0,2464 x^{2} R^{2}=0,95$

$\left(\right.$ AMVA) $Y_{1}=9,13-1,26 x-0,03 x^{2} R^{2}=0,97$

$(A A V A) Y_{2}=10,782-3,3029 x+0,267 x^{2} R^{2}=0,99$

$\left(\right.$ AMVP) $Y_{1}=9,18-1,22 x R^{2}=0,95$

$(\mathrm{AAVP}) \mathrm{Y}_{2}=10,68-3,98 \mathrm{x}+0,41 \mathrm{x}^{2} \mathrm{R}^{2}=0,91$

Período pós-colheita (Dias)

FIGURA 2 - Enrugamento (A) e Aceitação geral (B) em pitanga nos estádios de maturação-início de pigmentação (IP), vermelho-alaranjado (VA) e vermelho predominante $(\mathrm{VP})$ durante o armazenamento sob atmosfera modificada (AMY $\longrightarrow$ ) e ambiente $(\mathrm{AAY}-\longrightarrow$ ) a $10 \mathrm{e}$ a $14^{\circ} \mathrm{C}$ e à temperatura ambiente $\left(23 \pm 2^{\circ} \mathrm{C}\right)$. $\mathrm{O}$ limite 4 indica o nível de aceitação do fruto in natura pelo consumidor de pitanga. 
durante o armazenamento, evidenciando o efeito protetor da atmosfera modificada com relação à perda de água dos frutos (Ben-Yehoshua, 1987) e à manutenção de turgescência de pitangas (Fig. 2A). Segundo Kays (1997), quando as perdas de umidade, especialmente em frutos pequenos, atingem valores superiores a $5 \%$, podem surgir sintomas de enrugamento, os quais, mesmo não alterando a qualidade interna, prejudicam sua aparência, tornando-os inaceitáveis ao consumidor. A utilização da atmosfera modificada resultou, portanto, em maior vida útil de pitangas, enquanto os frutos sob AA se enrugaram rapidamente.

O uso de AM foi eficaz em manter a aparência, independentemente do estádio de maturação, principalmente quando sob refrigeração (Fig. 2B). Os frutos sob AM, pertencentes ao estádio IP mantidos em temperatura ambiente, apresentaram boa aparência até o $4^{\circ}$ dia de armazenamento. Para frutos colhidos no estádio vermelhoalaranjado (VA), a AM proporcionou a manutenção de boa aparência durante 3 e 6 e 8 dias, quando mantidos a $23 ; 14$ e $10^{\circ} \mathrm{C}$, respectivamente. Para o estádio vermelho predominante (VP), mantidos sob AM, a manutenção da qualidade dos frutos a $23 ; 14$ e $10{ }^{\circ} \mathrm{C}$ foi $4 ; 4$ e 7 dias, respectivamente. Portanto, a refrigeração prolongou a vida útil dos frutos quando comparados à temperatura ambiente, sobretudo para frutos no estádio VA e sob atmosfera modificada.

O uso de AM inibiu o desenvolvimento da cor de pitangas no estádio IP, mantidas sob refrigeração. Possivelmente, a elevação da concentração de $\mathrm{CO}_{2}$ e redução de $\mathrm{O}_{2}$ no interior da embalagem, associada à baixa temperatura de armazenamento, interferiu na atividade enzimática de modo a afetar o metabolismo dos pigmentos, como também foi verificado em morangos (Saez et al., 1999). A aparência dos frutos foi caracterizada pela cor alaranjada com tons leves de vermelho que predominou no final do armazenamento. Este comportamento pode estar associado à alteração da atmosfera que pode ter afetado o metabolismo de degradação da clorofila e o pleno desenvolvimento dos carotenóides.

Pitangas no estádio vermelho predominante (VP), mantidas sob AA a $23^{\circ} \mathrm{C}$, encontravam-se visualmente comprometida após o $2^{\circ}$ dia. A perda da qualidade, provavelmente, se deu em função da elevada perda de água sofrida pelos frutos, da oxidação de clorofila e carotenóides, a qual proporcionou a cor vermelho-escura nos frutos, bem como da infecção fúngica, uma vez que frutos maduros possuem menor resistência de seus tecidos, sendo mais vulneráveis à deterioração durante o armazenamento (Saez et al., 1999). A maioria dos processos que conduzem à perda de qualidade estão relacionados à temperatura $\mathrm{e}$ à umidade relativa do ambiente de armazenamento (Jaime et al., 2001). A temperatura determina a quantidade de vapor de água necessária para saturar a atmosfera, afetando a fisiologia do produto e o desenvolvimento de microrganismos (Kays, 1997). Por esta razão, o controle da temperatura é, provavelmente, o fator mais importante na redução da deterioração do produto armazenado (Kader, 1986). Pitangas são frutos que possuem atividade metabólica elevada (Santos et al., 2003) e, conseqüentemente, vida pós-colheita curta. O uso de refrigeração foi um fator determinante para o prolongamento da vida útil de pitangas. Quando a refrigeração foi associada à AM, obteve-se redução da perda de peso e do enrugamento (Beaudry, 2004), mantendose a turgidez dos frutos por mais tempo.

\section{CONCLUSÕES}

1. A elevada perda de massa ocorrida em frutos mantidos à temperatura ambiente foi o fator limitante na manutenção da vida útil pós-colheita de pitangas mantidas sob atmosferas ambiente (AA) e modificada (AM), com pronunciado enrugamento.
2. A incidência de fungos foi a maior limitação do armazenamento sob atmosfera modificada.

3. O uso de atmosfera modificada para frutos vermelhoalaranjados (VA) mantidos a $10{ }^{\circ} \mathrm{C}$ foi a condição mais eficiente na conservação de pitangas, possibilitando a manutenção da turgidez, da qualidade, da integridade e aparência atrativas durante oito dias de armazenamento.

\section{AGRADECIMENTOS}

Os autores agradecem ao CNPq pelo suporte financeiro para a realização deste experimento.

\section{REFERÊNCIAS}

AHMADI, H.; BIASI, W.V.; MITCHAM, E.J. Control of brown rot decay of nectarines with $15 \%$ carbon dioxide atmosphere. Journal American Society Horticulture Science, Alexandria, v.124, n.5, p. 708-712, 1999

BEAUDRY, R.M. Modified atmosphere packaging. In: HARDENBURG, R.E; WATADA, A.E.; WANG, C.Y.; GROSS, K. The commercial storage of fruits, vegetables, and florists, and nursery stocks. Washington, D.C.: US Departament of Agriculture, 2004, p. 456567.

BEN-YEHOSHUA, S. Transpiration, water stress and gas exchange. In: WEICHMANN, J. Postharvest physiology of vegetables, New York: Marcel Dekker, 1987. p.113-170.

GRIERSON, W.; WARDOWSKI, W. F. Relative humidity effects on the post-harvest life in fruits and vegetables. HortScience, Alexandria, v.13, n.5, p.22-26, 1978.

JAIME, P.; SALVADOR, M.L.; ORIA, R. Respiration rate of sweet cherries: 'Burlat', Sunburst' and 'Sweetheart' cultivars. Journal of Food Science, Chicago, v.66, n.1, p. 43-47, 2001.

KADER, A. A. Biochemical and physiological basis for effects of controlled and modified atmospheres on fruits and vegetables. Food Technology, Chicago, v.40, n.5, p. 99-104, 1986.

KAYS, S. J. Postharvest physiology of perishable plant products. Athens: Exonn Press, 1997. 532p.

MEIER, S.; ROSENBERG, I.; FALLIK, E. Improvement of postharvest Keeping quality and color development of bell peper by packaging with polyethylene bags at a reduced temperature. Postharvest Biology and Technology, Amsterdan, v.5, p.303-309, 1995.

MELO, E. A.; LIMA, V. L. A. G. de; NASCIMENTO, P. P. do. Temperatura no armazenamento de pitanga. Scientia Agrícola, Piracicaba, v.57, n.4, p. 629-634, 2000

MORRIS, L. L. Chilling injury of horticultural crops: an overview. HortScience, Alexandria, v. 17, n.2, p.161-162, 1982.

SAEZ, C; OLÍAS, P. R.; OLÍAS, J. M. Quality of strawberries packed with perforated polypropylene. Journal of Food Science, Chicago, v.64, n.4, p.748-751, 1999.

SANTOS, A. F.; SILVA, S. M.; MENDONÇA, R. M. N.; SILVA, M. S.; ALVES, R. E.; FILGUEIRAS, H. A. C. Alterações fisiológicas durante a maturação de pitanga (Eugenia uniflora L.) Proceedings of the Interamericana Society for Tropical Horticulture, Miami, v.46, p.5257, 2003.

SANTOS, A. F.; SILVA, S.M.; MENDONÇA, R.M. N.; ALVES, R. E.; FILGUEIRAS, H. A. C. Maturation and ripening changes in Suriname Cherry (Eugenia uniflora L.) fruits. Proceedings of the Interamericana Society for Tropical Horticulture, Miami, v.45, p.4547, 2002. 